\title{
Hinode extreme-ultraviolet imaging spectrometer observations of a limb active region
}

\author{
B. O’Dwyer ${ }^{1}$, G. Del Zanna ${ }^{1}$, H. E. Mason ${ }^{1}$, A. C. Sterling ${ }^{2, \star}$, D. Tripathi ${ }^{1}$, and P. R. Young ${ }^{3,4}$ \\ 1 Department of Applied Mathematics and Theoretical Physics, Wilberforce Road, Cambridge, CB3 OWA, UK \\ e-mail: [B.0-Dwyer;G.Del-Zanna;H.E.Mason; D.Tripathi]@damtp.cam.ac.uk \\ 2 NASA, Marshall Space Flight Center, Huntsville, Alabama, USA \\ e-mail: alphonse.sterling@nasa.gov \\ 3 Space Science Division, Naval Research Laboratory, Washington, DC 20375, USA \\ ${ }^{4}$ George Mason University, 4400 University Drive, Fairfax, VA 22030, USA
}

Received 15 June 2009 / Accepted 29 July 2010

\begin{abstract}
Aims. We investigate the electron density and temperature structure of a limb active region.

Methods. We have carried out a study of an active region close to the solar limb using observations from the Extreme-ultraviolet Imaging Spectrometer (EIS) and the X-ray telescope (XRT) on board Hinode. The electron density and temperature distributions of the coronal emission have been determined using emission line intensity ratios. Differential emission measure (DEM) analysis and the emission measure (EM) loci technique were used to examine the thermal structure of the emitting plasma as a function of distance from the limb.

Results. The highest temperature and electron density values are found to be located in the core of the active region, with a peak electron number density value of $1.9 \times 10^{10} \mathrm{~cm}^{-3}$ measured using the Fe XII $186.887 \AA$ to $192.394 \AA$ line intensity ratio. The plasma along the line of sight in the active region was found to be multi-thermal at different distances from the limb. The EIS and XRT DEM analyses appear to be in agreement in the temperature interval from $\log T=6.5-6.7$.

Conclusions. Our results provide new constraints for models of coronal heating in active regions.
\end{abstract}

Key words. Sun: activity - Sun: atmosphere - Sun: corona - Sun: UV radiation

\section{Introduction}

An understanding of the mechanism responsible for heating the solar corona is one of the major outstanding problems of solar physics. Before various coronal heating models can be fully evaluated, it is critically important to determine quantitative physical properties of coronal features from observations. The study of active regions is of particular relevance to the coronal heating problem as they act as the locations of the most pronounced heating processes.

One possible solution to the coronal heating problem is impulsive heating of coronal loops by nanoflares occurring within unresolved strands (Parker 1988; Cargill 1994; Cargill \& Klimchuk 1997). A number of observations that are inconsistent with static models, such as the density excess of $1 \mathrm{MK}$ loops (Aschwanden et al. 2001), can be explained by nanoflare heating (Klimchuk 2006). However, the frequent occurrence of nanoflares would require the presence of plasma with temperatures in excess of $5 \mathrm{MK}$ in the corona (Cargill 1994). The detection has proved elusive, perhaps partly because the intensity of the hot emission is expected to be very weak (Klimchuk et al. 2008).

Diagnostic studies of eclipse observations (see Gabriel \& Jordan 1975), early X-ray observations (Vaiana 1976), Skylab observations (Webb 1981) and SOHO CDS observations (Mason et al. 1999; Del Zanna \& Mason 2003; Milligan et al. 2005;

* Current location: JAXA/ISAS, Hinode Group, Yoshinodai 3-1-1, Sagamihara, Kanagawa, 229-8510, Japan.
Tripathi et al. 2006) all indicate that active regions have a hot, dense core. Sterling et al. (1997) and Sterling (1997) used data from the Bragg Crystal Spectrometer (BCS) instrument on the Yohkoh satellite to study the temperature structure of active regions with height, and found that the highest temperatures occurred at the base of the active region, with a fall off of temperatures at greater heights. BCS however did not have any spatial resolution, and those studies inferred the height structure of the temperature by using the solar limb as an occulting edge for a single active region which rotated around the disk and behind the limb. Sterling et al. (1999) verified BCS findings by measuring spectral-scans from the Coronal Diagnostic Spectrometer (CDS) on the SOHO, integrated over different heights of an active region. Others have also investigated the thermal structure of active regions with various instruments (e.g., Pye et al. 1978; Schadee et al. 1983; Watanabe et al. 1995; Yoshida \& Tsuneta 1996; Warren \& Winebarger 2003).

In this paper we have used the Extreme-ultraviolet Imaging Spectrometer (EIS; Culhane et al. 2007) on board Hinode (Kosugi et al. 2007) to study a limb active region, in order to determine the electron density and temperature structure, as well as the variation in emission measure, as a function of distance from the limb. EIS provides the opportunity to study individual spectral emission lines, allowing a precise definition of the electron density and temperature distribution with a high spatial resolution. In Sect. 2 we provide new Hinode/EIS observations and our data analysis techniques. Our results are presented in Sect. 3, with our conclusions in Sect. 4. 

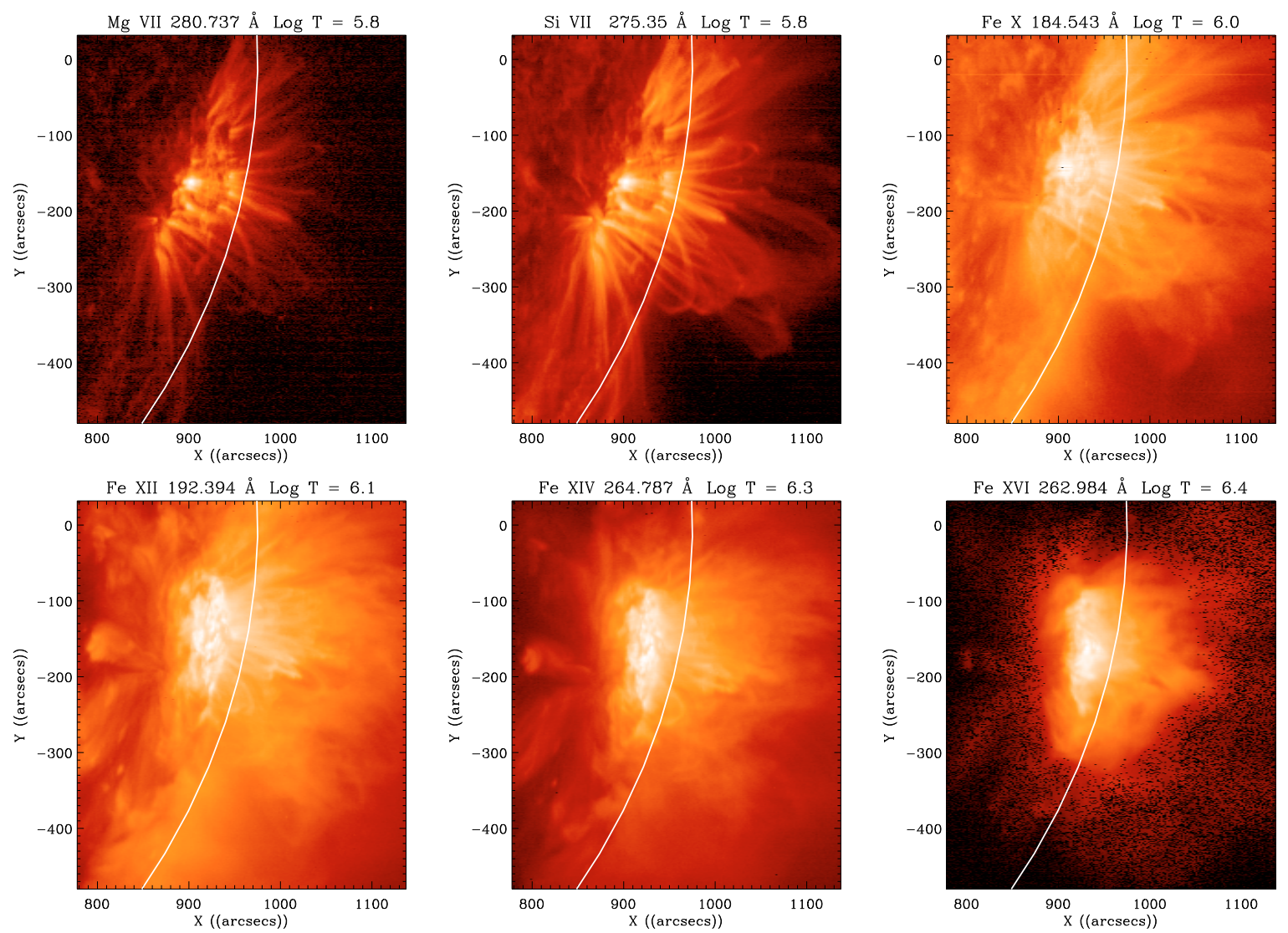

Fig. 1. EIS Intensity maps from a sample of emission lines formed over the temperature range $\log T=5.8-6.4$.

\section{Observations and data analysis}

EIS is an off-axis spectrometer of paraboloid design with a focal length of $1.9 \mathrm{~m}$ and mirror diameter of $15 \mathrm{~cm}$. It consists of a multi-toroidal grating which disperses the spectrum on two different detectors. The first detector covers the wavelength range 170-211 $\AA$ and the second covers 246-292 A providing observations of solar corona and upper transition region (TR) emission lines. EIS has four slit options available (of widths $1^{\prime \prime}, 2^{\prime \prime}$, $40^{\prime \prime}$ and $\left.266^{\prime \prime}\right)$. EIS provides monochromatic images at a high cadence using a slot (defined as the $40^{\prime \prime}$ or $266^{\prime \prime}$ slit widths). High spectral resolution images can be obtained by rastering with a slit.

Observations were made on 17 December, 2007 using EIS. The target of opportunity was an active region close to the solar limb, with the core still on the disk. The observation sequence we designed (CAM_AR_LIMB) used the $2^{\prime \prime}$ slit with an exposure time of $45 \mathrm{~s}$. The EIS raster used in this analysis started at 10:42:20 UT. The raster was 360" wide, used a slit length of $512^{\prime \prime}$ and lasted for $2.5 \mathrm{~h}$.

The data were first processed using the standard processing routine eis_prep.pro provided in SolarSoftWare software package (Freeland \& Handy 1998). This routine removes the dark current and CCD pedestal, as well as hot pixels and cosmic ray hits. A line fitting routine, written by one of us (GDZ) using cfit (Haugan 1997), was applied to all of the lines at each pixel in the EIS raster. Instrumental effects such as the EIS slit-tilt and wavelength shifts due to spacecraft orbital variation have been compensated for. To estimate the orbital variation it is necessary to select a quiet region in the raster. The bottom 50 pixels in the raster, which are furthest away from the active region, were selected for this purpose. By studying the average variations of
Table 1. XRT data for the off-limb area.

\begin{tabular}{cccc}
\hline \hline Filter & $\begin{array}{c}\text { Long Exp. } \\
(\mathrm{s})\end{array}$ & $\begin{array}{c}\text { Short Exp. } \\
(\mathrm{s})\end{array}$ & $\begin{array}{c}\text { Obs. Flux } \\
\left(\mathrm{DN} \mathrm{s}^{-1} \text { pixel }^{-1}\right)\end{array}$ \\
\hline Al_poly & 0.72 & 0.06 & 2900 \\
C_poly & 1.44 & 0.09 & 2020 \\
Be_thin & 5.80 & 0.13 & 635 \\
Be_med & 2.05 & & 111 \\
Ti_poly & 2.90 & 0.06 & 1330 \\
Al_thick & 23.1 & & 4.13 \\
Be_thick & 32.8 & & 0.12 \\
Al_poly-Ti & 4.10 & 0.13 & 932 \\
\hline
\end{tabular}

the line centroids, the effect of orbital variation can be determined. Intensity maps are displayed in Fig. 1 from a sample of emission lines formed over the temperature range log $T[\mathrm{~K}]=$ 5.8-6.4. It can be clearly seen that the low temperature emission is better defined in discrete structures than the high temperature emission. This has been observed previously by Tripathi et al. (2009). The diffuse background emission seen in the coronal ions Fe X-Fe XIV is real and not a function of the way the data are displayed. A list of the emission lines used in the present study is given in Table 1.

Over the duration of the EIS raster, observations of the active region were also made using the X-ray Telescope (XRT; Golub et al. 2007) on board Hinode. An XRT image of the active region observed with the C_poly filter is shown in Fig. 2. We note the similarity in the observed structures in the Fe XVI $262.984 \AA$ intensity map in Fig. 1 and the XRT image in Fig. 2. 


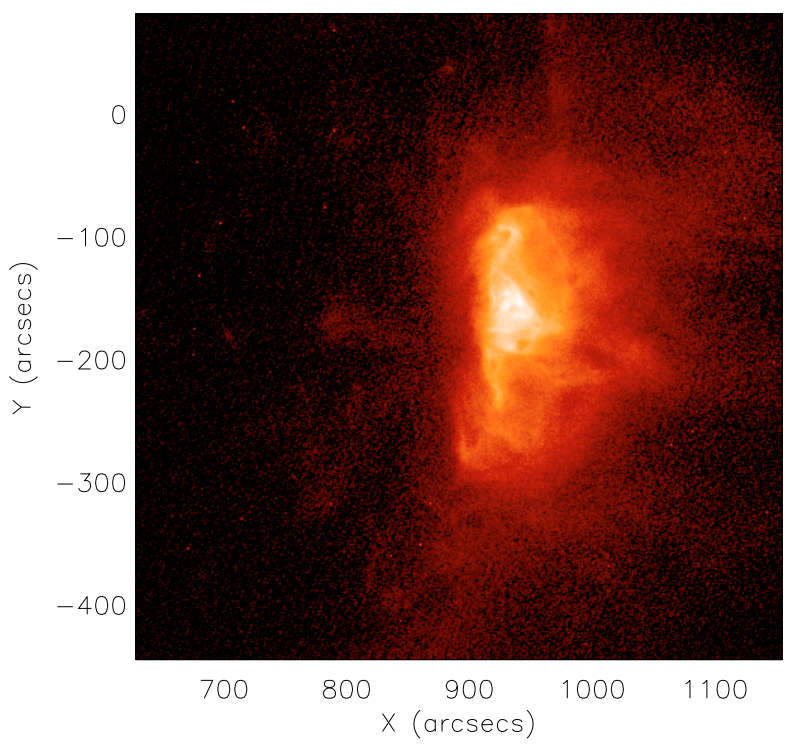

Fig. 2. XRT image observed with the C_poly filter.

Under the assumption that a plasma is optically thin and in ionization equilibrium, the observed intensity of a spectral emission line can be expressed as

$I_{\mathrm{ob}}=A(\mathrm{z}) \int_{T_{\mathrm{e}}} G\left(T_{\mathrm{e}}, N_{\mathrm{e}}\right) \varphi\left(T_{\mathrm{e}}\right) \mathrm{d} T_{\mathrm{e}}$

where $A(\mathrm{z})$ is the elemental abundance, $T_{\mathrm{e}}$ is the electron temperature, and $N_{\mathrm{e}}$ is the electron density. The contribution function, $G\left(T_{\mathrm{e}}, N_{\mathrm{e}}\right)$, contains the relevant atomic parameters for each line and can be obtained using equilibrium ionization balance calculations. For this purpose we have used CHIANTI, v5.2 (Dere et al. 1997; Landi et al. 2006). The quantity $\varphi\left(T_{\mathrm{e}}\right)$ is known as the differential emission measure which is defined as

$\varphi\left(T_{\mathrm{e}}\right)=N_{\mathrm{e}}{ }^{2} \frac{\mathrm{d} h}{\mathrm{~d} T_{\mathrm{e}}}$

where $h$ is the column height along the line-of-sight (LOS).

To determine the average temperature along the LOS for a given point we have used the EM-loci method (see Del Zanna et al. 2002, and references therein). This method involves plotting the ratio of $I_{\mathrm{ob}} /\left(A(\mathrm{z}) G\left(T_{\mathrm{e}}, N_{\mathrm{e}}\right)\right)$ for each line that decays to the ground state as a function of temperature. The loci of these curves constrain the shape of the emission measure distribution. In the case of an isothermal plasma, the curves should intersect at a common point. If the plasma is not isothermal, then it is necessary to perform a DEM analysis in order to obtain the distribution of plasma along the LOS. To evaluate the DEM distributions, we used an application of the "maximum entropy method", as described in Monsignori Fossi \& Landini (1991) and implemented by Del Zanna (1999). The DEM function is assumed to be a cubic spline with a limited number of selected "mesh" points, covering the temperature range where observational constraints (spectral lines) are present. The fitting program allows one to search for the best solution for each mesh point.

\section{Results}

\subsection{Densities}

The areas contained within the annular sectors marked in Fig. 3 show the locations of an active region strip and a quiet sun region (selected to study the background emission). The variation

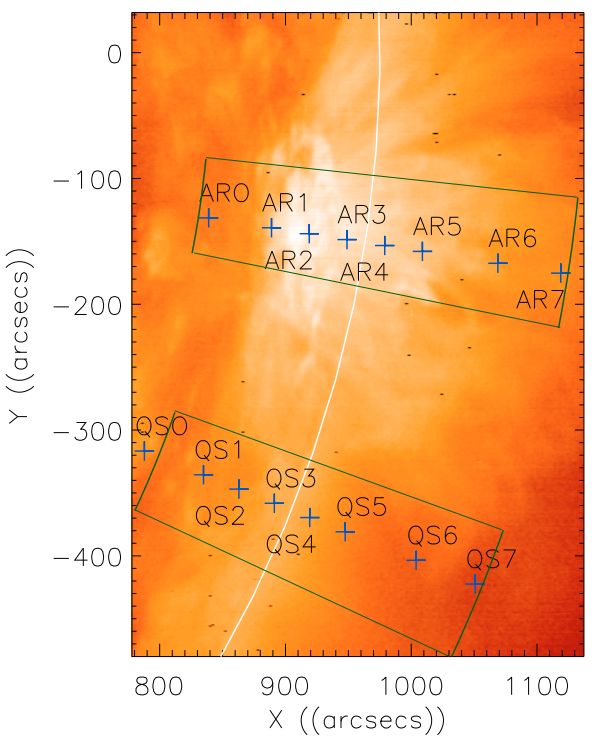

Fig. 3. EIS Fe XI Intensity map showing the location of an active region strip and a quiet sun region in green. Also included are the positions at which the DEM curves were constructed shown in blue.

in line intensity as a function of distance from the limb was investigated for a sample of emission lines in the data set. This was achieved by calculating the average intensity in ten-pixelwide annular sectors at fixed distances from the limb for each emission line. Figures 4 and 5 show plots of average line intensity as a function of distance from the limb for a sample of high and low temperature emission lines respectively, in the active region strip (Figs. 4a, 5a) and quiet sun region (Figs. 4b, 5b) shown in Fig. 3. We note that in the AR the Fe XVI $262.984 \AA$ line falls off more sharply above the limb than the lower temperature lines. We also note the sharp fall off of the transition region lines above the limb in the quiet sun region. The variation in intensity as a function of distance from the limb is qualitatively different for the Fe XIII 203.828 $\AA$ and 202.044 $\AA$ lines. This is due to the density sensitivity between the lines. We note that the comparison of the intensity variation with height of densityinsensitive emission lines from the same ion is a useful method of identifying line blends.

Electron density maps were generated using the densitysensitive line intensity ratios of Fe XIV 264.787 $\AA$ to $274.204 \AA$, Fe XIII 203.826 ̊ to $202.044 \AA$, Fe XII $186.887 \AA$ to $192.394 \AA$, Si X 258.372 $\AA$ to $271.992 \AA$ and Mg VII $278.395 \AA$ to $280.737 \AA$. The method used for electron density diagnostics is described in detail in Young et al. (2007, 2009). The theoretical ratios were calculated using CHIANTI, v5.2. Figure 6 displays a sample density map created using the Fe XIII $203.826 \AA$ to $202.044 \AA$ ratio. The density maps created from the other ions were qualitatively similar. The highest electron density values are found to be located in the core of the active region. A peak value of $1.9 \times 10^{10} \mathrm{~cm}^{-3}$ was measured using Fe XII. These results are similar to those of Tripathi et al. (2008a,b) who studied Hindoe/EIS observations of an on-disk active region. The electron density values decrease with height above the limb, with values of $\sim 3.8 \times 10^{8} \mathrm{~cm}^{-3}$ found at a height of $150^{\prime \prime}$ $(109000 \mathrm{~km})$ above the limb. Figure 7 displays the average electron density along the line of sight in the active region strip, calculated for different ions, plotted as a function of distance from the solar limb. One sigma uncertanties on the line intensities have been translated to errors on the derived densities. 


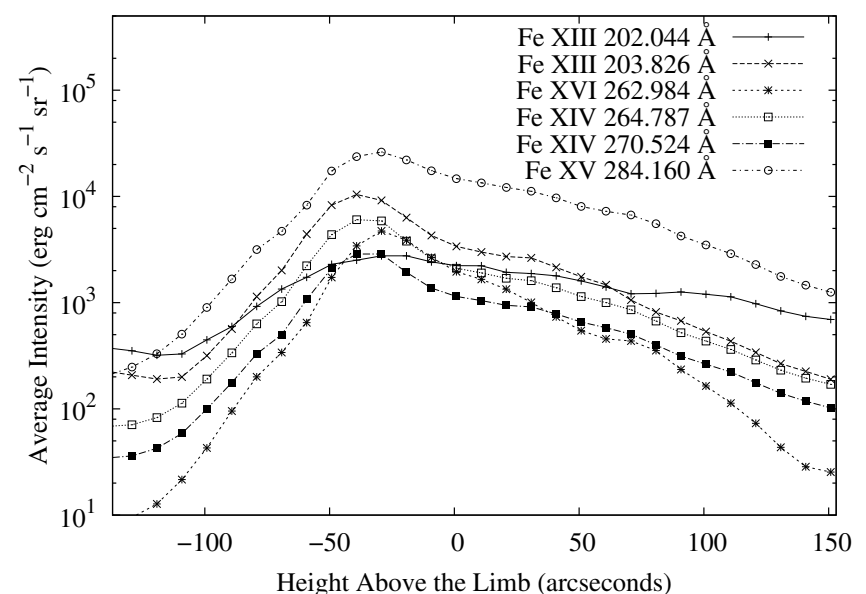

(a) Active region strip

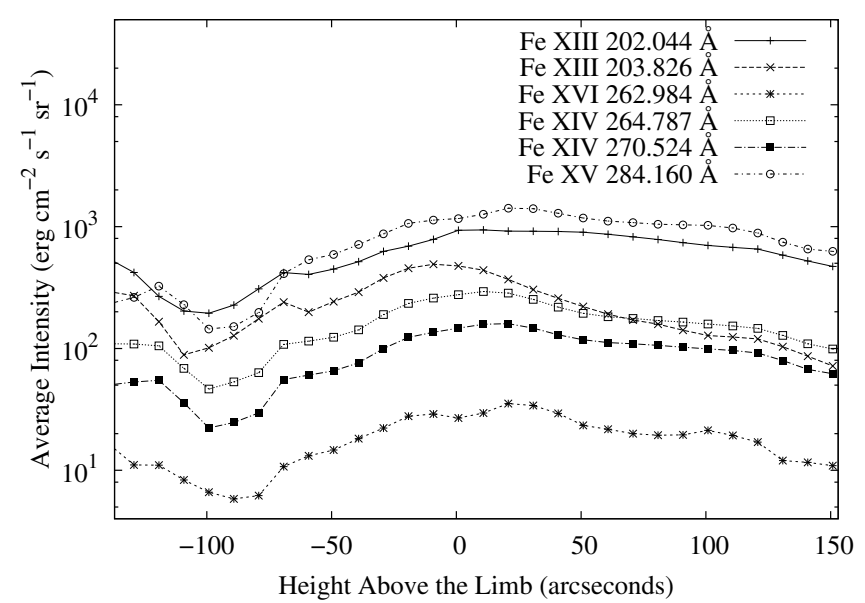

(b) Quiet sun region

Fig. 4. Plots of average line intensity as a function of distance from the limb for high temperature emission lines in the active region strip and quiet sun region displayed in Fig. 3.

The uncertainties in the theoretical ratios are more difficult to estimate. Individual ions are discussed below. We note that the Mg VII intensities were weak and reliable counts could not be obtained above the limb. In Fig. 7 error bars on the Mg VII number densities are omitted in order to improve the clarity of the figure. We estimate typical uncertainties on the electron densities derived using $\mathrm{Mg}$ VII as approximately $\pm 2.0 \times 10^{9} \mathrm{~cm}^{-3}$. We note from Fig. 7 that the electron densities derived from Fe XII seem to be somewhat higher than those obtained from other ions. Young et al. (2009) found that Fe XII gave larger densities than Fe XIII in a study involving active regions, as did Tripathi et al. (2008b) in high density regions. The discrepancies between different ions are difficult to explain. We note that the atomic data for Si X and Fe XIII have recently been updated, as described in Liang et al. (2009) and Storey \& Zeippen (2010). The most recent atomic data for Fe XII is discussed in Storey et al. (2005). Further checks on line blending are in progress.

\subsection{Temperatures}

Temperature maps were generated using density-insensitive emission line ratios from adjacent ionization states of iron. The temperature range over which this diagnostic is sensitive corresponds to the overlap of the contribution functions from the

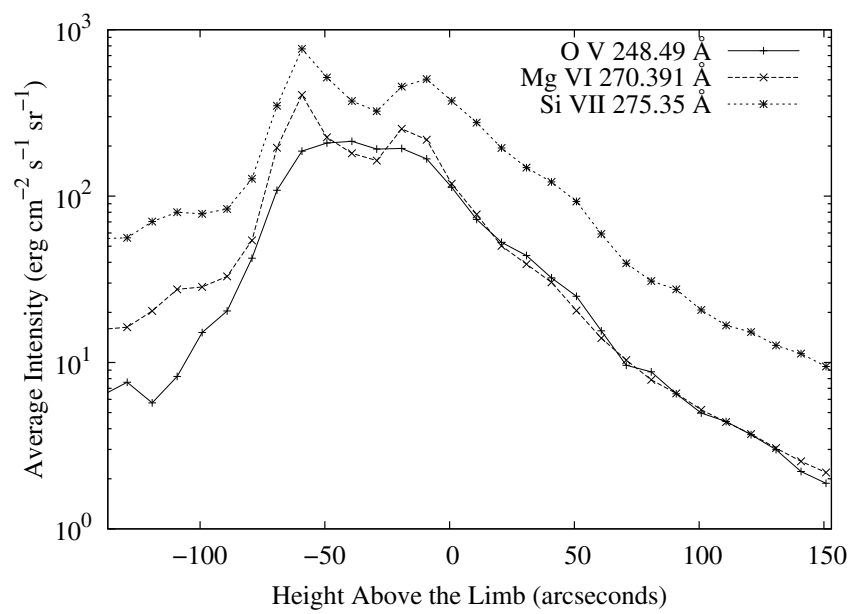

(a) Active region strip

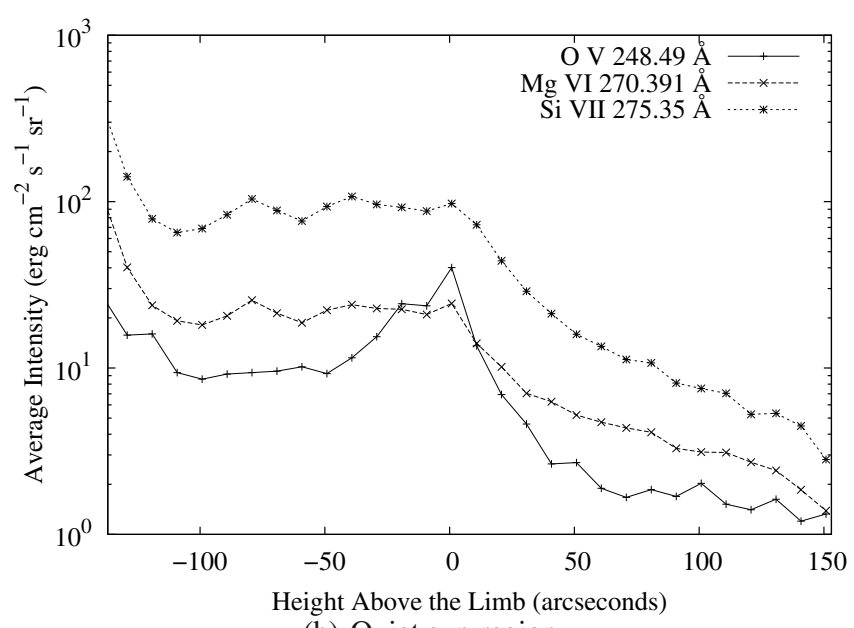

(b) Quiet sun region

Fig. 5. Plots of average line intensity as a function of distance from the limb for low temperature emission lines in the active region strip and quiet sun region displayed in Fig. 3.

individual lines. Figure 8 displays the temperature range over which the ion fractions of the Fe XV and Fe XVI ions overlap. A temperature map created using the line intensity ratio of Fe XVI $262.984 \AA$ to Fe XV 284.160 $\AA$ is shown in Fig. 9, sensitive over the temperature range $\log T=6.2-6.7$. It should be noted that this method only provides a crude indication of the temperature, since it assumes that the plasma along the line of sight is isothermal. Nevertheless, the image shows the locations of the hottest areas (in the core) and provides values in agreement with the DEM findings (see Sect. 3.3). The highest electron temperature values $(\log T=6.7)$ are found to be located in the core of the active region.

Due to its importance for coronal heating models involving nanoflares, the coronal emission at temperatures above $5 \mathrm{MK}$ was specifically examined. Among the lines sensitive to such high temperatures the strong Fe XVII 254.87 $\AA$ line was not present in our study. Instead the weak Fe XVII $275.55 \AA$ line (see Del Zanna \& Ishikawa 2009) was fitted by summing the intensity into bins of nine pixels and applying a three Gaussian fit to the lines in the window (Si VII 275.354 $\AA$, Fe XVII 275.55 Si VII $275.668 \AA$ ). An intensity map generated using the Fe XVII emission line is displayed in Fig. 10a. 
B. O'Dwyer et al.: Hinode EIS observations of a limb active region

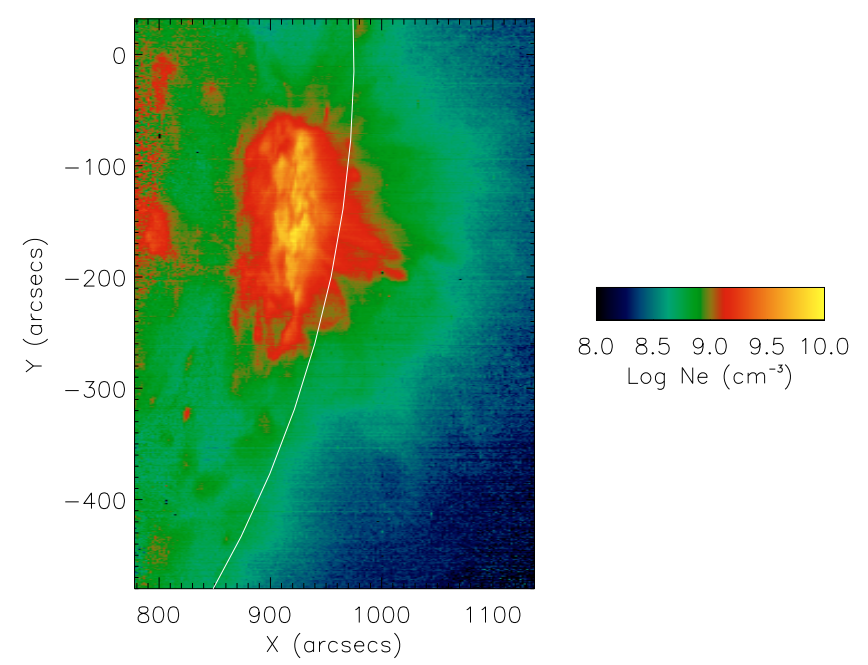

Fig. 6. Electron number density map created using the Fe XIII $203.826 \AA$ to $202.044 \AA$ A ratio.

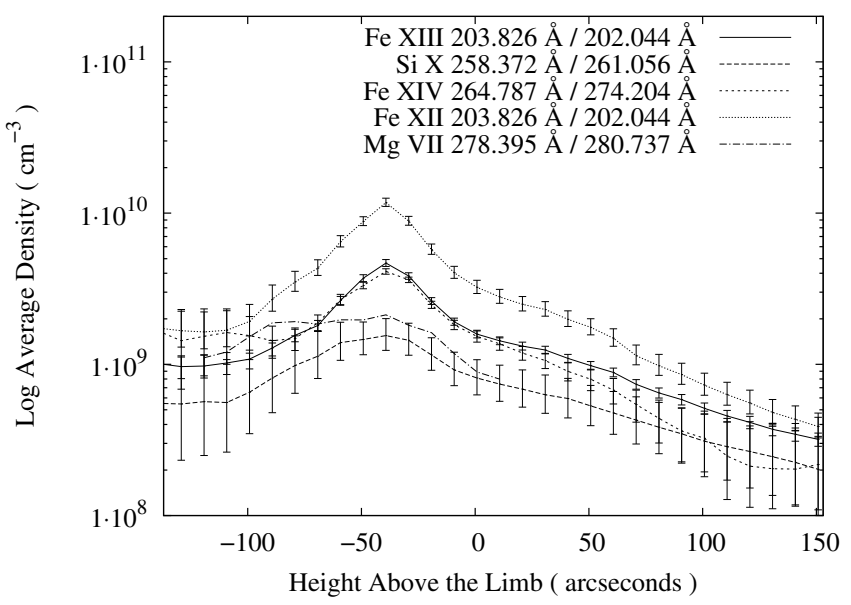

Fig. 7. Plots of electron number density as a function of distance from the limb in the active region strip displayed in Fig. 3. The error bars on the electron density values are derived from the one sigma uncertainties on the observed intensities. See text for discussion of Mg VII error bars.

An attempt was also made to resolve the blended Ca XVII $192.820 \AA$ line. The line is blended with the Fe XI 192.813 $\AA$ line, several O V lines, as well as the weak Fe XI $192.90 \AA$ line. The Fe XI 192.813 $\AA$ blend was removed by using a value of 0.27 for the ratio of Fe XI $192.813 \AA$ to Fe XI 188.216 $\AA$ obtained off-limb in the quiet sun region. Using a similar approach Ko et al. (2009) obtained a value of 0.26 for this ratio. At the AR3 position shown in Fig. 3 the measured intensity of the Fe XI 188.216 $\AA$ line is $2610 \mathrm{erg} \mathrm{cm}^{-2} \mathrm{~s}^{-1} \mathrm{sr}^{-1}$, giving an Fe XI $192.813 \AA$ intensity of $705 \mathrm{erg} \mathrm{cm}^{-2} \mathrm{~s}^{-1} \mathrm{sr}^{-1}$. The $\mathrm{O} V$ blends were accounted for by adopting an $\mathrm{O} V$ electron number density of $1.0 \times 10^{9} \mathrm{~cm}^{-3}$ to obtain a value of 1.37 for the ratio of the blended O V lines to the O V $248.49 \AA$ line from CHIANTI. At the AR3 position the measured intensity of the $\mathrm{O} \mathrm{V} 248.49 \AA$ line is $211 \mathrm{erg} \mathrm{cm}^{-2} \mathrm{~s}^{-1} \mathrm{sr}^{-1}$, giving a value of $289 \mathrm{erg} \mathrm{cm}^{-2} \mathrm{~s}^{-1} \mathrm{sr}^{-1}$ for the contribution of the $\mathrm{O} \mathrm{V}$ lines to the total blend. The Fe XI $192.90 \AA$ line can be estimated by using the branching ratio of Fe XI 192.90 ̊ to Fe XI 188.30 ̊. The value of this ratio is 0.016 . At the AR3 position the estimated value of the Fe XI $192.90 \AA$ line was $35 \mathrm{erg} \mathrm{cm}^{-2} \mathrm{~s}^{-1} \mathrm{sr}^{-1}$. The measured intensity of the total blend was $2330 \mathrm{erg} \mathrm{cm}^{-2} \mathrm{~s}^{-1} \mathrm{sr}^{-1}$.

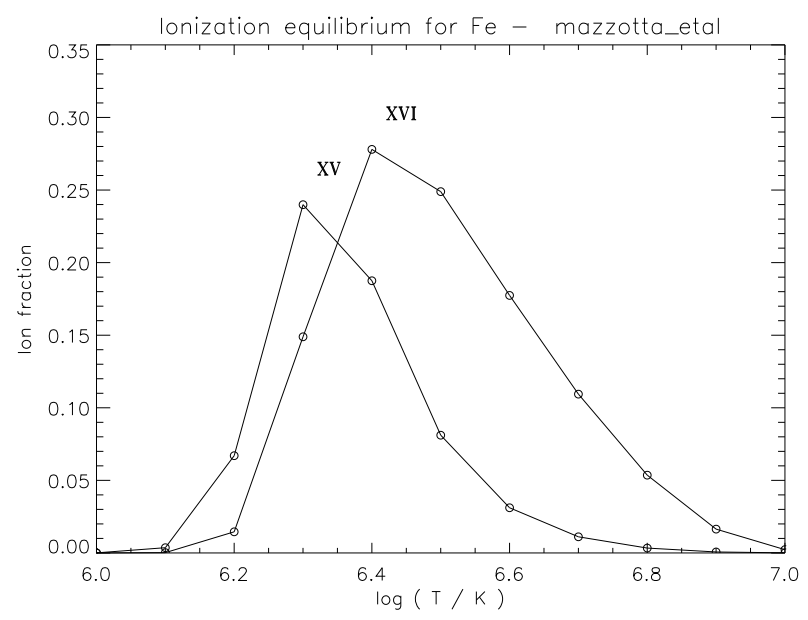

Fig. 8. Ion fractions as a function of temperature for Fe XVI and Fe XV using the ionization equilibrium calculations of (Mazzotta et al. 1998).

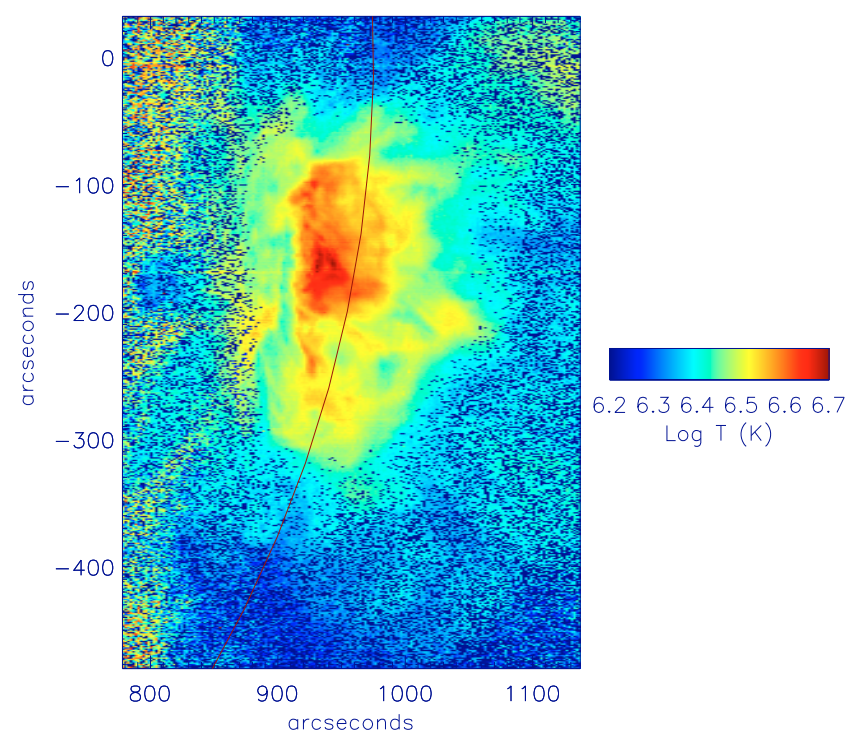

Fig. 9. Electron temperature map created using the line intensity ratio of Fe XVI 262.984 ̊̊ to Fe XV 284.160 ̊.

We consider the contribution of the Fe XI $192.90 \AA$ line to the total blend to be negligible. A value of $1340 \mathrm{erg} \mathrm{cm}^{-2} \mathrm{~s}^{-1} \mathrm{sr}^{-1}$ was calculated for the intensity of the Ca XVII $192.820 \AA$ line at the AR3 position. Figure 10b displays an intensity map generated using the derived Ca XVII $192.820 \AA$ line. For each pixel in the map the derived $\mathrm{Ca}$ XVII intensity is displayed if it contributes more than $10 \%$ to the total blend.

The temperatures of formation of the Fe XVII and Ca XVII ions are $\log T=6.6$ and 6.7 respectively. We would therefore expect emission from these ions to trace out plasma at similar temperatures. The similarity between the structure of the coronal emission in the Fe XVII and Ca XVII intensity maps, shown in Fig. 10, indicates that the Ca XVII line has been correctly deblended. Ko et al. (2009) have also carried out a detailed study of the Ca XVII line with Hinode/EIS. They find that the Ca XVII line can only be reliably deblended if its intensity is greater than ten percent of the total line blend. In our study the contribution of the Ca XVII line to the total blend is greater than ten percent for $\mathrm{AR} 2-\mathrm{AR} 4$. Consistent with the temperatures derived from the Fe XVI 262.984 $\AA$ to Fe XV 284.160 $\AA$ ratio, our Fe XVII and Ca XVII maps also indicate that the hottest 

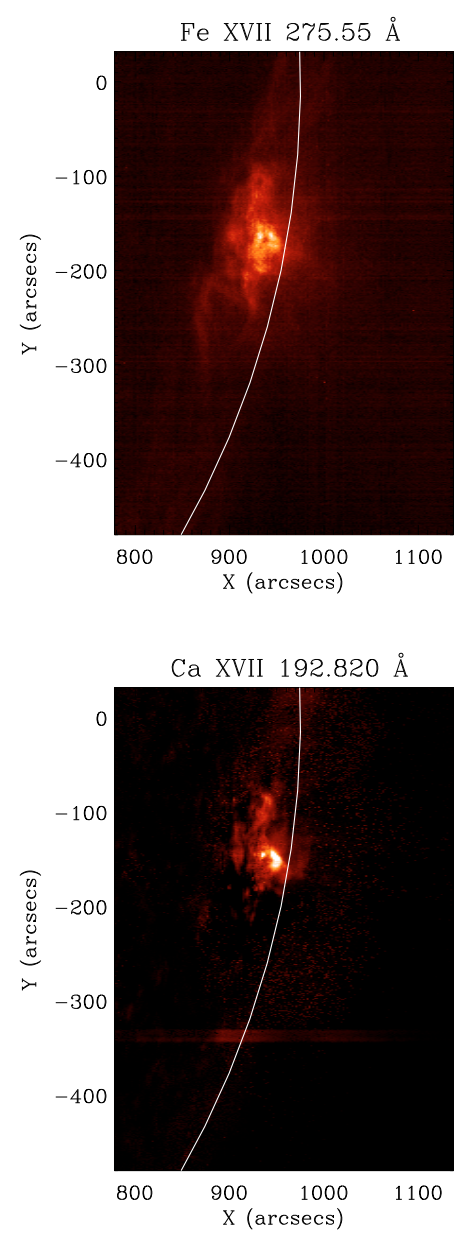

Fig. 10. EIS Intensity maps of high temperature emission lines. Figure 10a (top) Fe XVII. Figure 10b (bottom) Ca XVII.

portions of the active region are localized at low heights in the active region core, while further out the emission from hot material is much weaker. Moreover, the Ca XVII map indicates that for the regions where $\mathrm{Ca}$ XVII is definitely present, i.e. where it contributes more than ten percent to the total blend, the hottest parts (brightest in the map of Fig. 10b) seem to be concentrated in specific locations within the core.

\subsection{Emission measure}

The emission measure (EM) loci technique and a differential emission measure (DEM) analysis were used to examine the thermal structure of the emitting plasma as a function of distance from the limb. EM loci plots were constructed at points across the active region shown as blue crosses in Fig. 3. At these positions the intensity of each emission line was summed in a three by three pixel area. A sample EM loci plot generated at the AR3 point is displayed in Fig. 11. It can be seen that the plasma along the line of sight is not isothermal at this position. The plasma along the line of sight was found to be multithermal at all of the positions AR0-AR7 based on the EM loci plots.

DEM curves were generated at points across the active region strip and quiet sun region. The positions at which the DEM curves were created are shown as crosses in Fig. 3. The density values at these positions, calculated from the Fe XII $186.887 \AA$ to $192.394 \AA$ ratio, were used to create the DEM curves. The emission lines used to constrain the

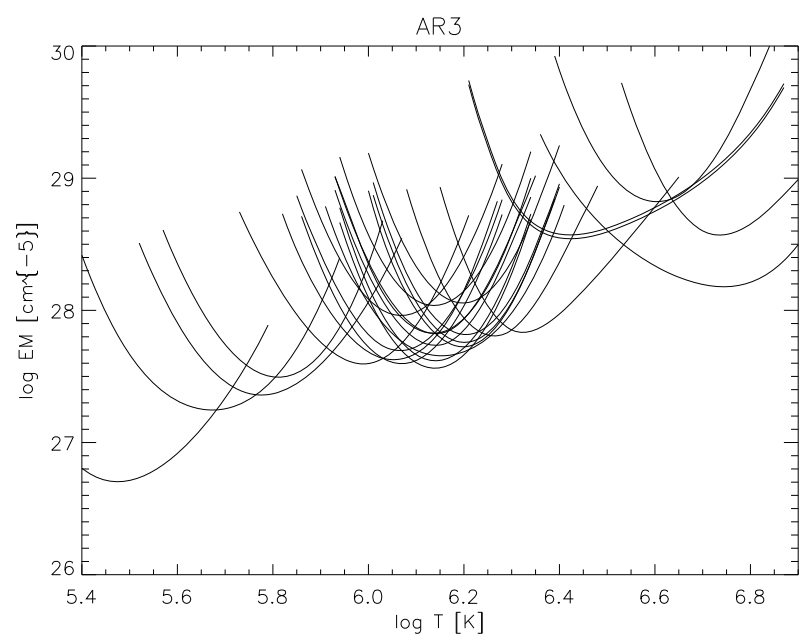

Fig. 11. EM loci plot constructed at the AR3 position shown in Fig. 3.

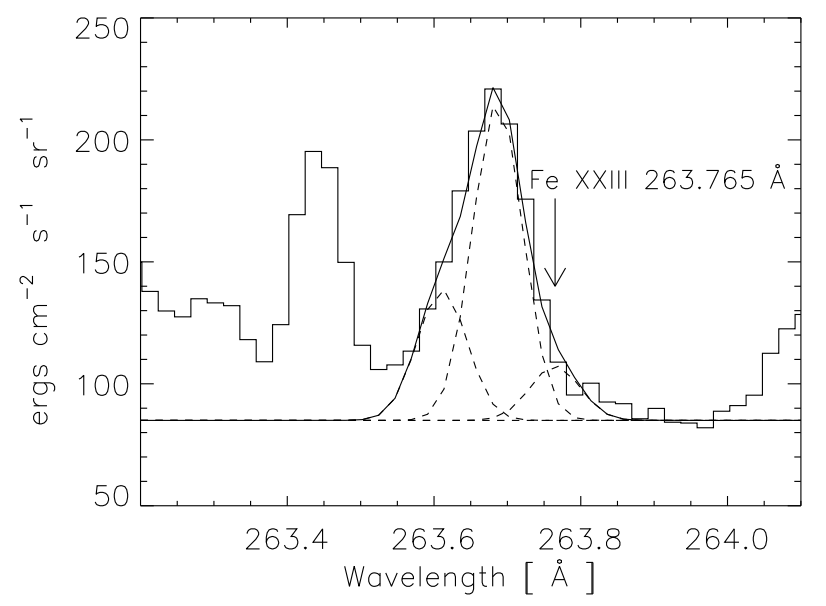

Fig. 12. Spectrum from the off-limb area in Fig. 16 showing the Fe XXIII $263.765 \AA$ line, blended with two unidentified lines.

DEM curves are listed in Table 1. We used the solar coronal abundance values of Feldman et al. (1992) and the ionization equilibrium calculations of Mazzotta et al. (1998). We note that the Fe XXIII $263.765 \AA$ line is blended with two unidentified transitions and is included in the DEM curves as an upper limit, indicated with an arrow. For positions AR1-AR5 a blended line was resolvable at the Fe XXIII 263.765 ^ wavelength, and in each case was fitted with three Gaussian functions. Figure 12 shows a spectrum, obtained from the off-limb area in Fig. 16, of the blended Fe XXIII $263.765 \AA$ line. The wavelengths have been calibrated using known unblended lines from the same spectral window. The intensity value derived from the Fe XXIII $263.765 \AA$ line fitting was used as an upper limit. At the AR and QS positions where a line could not be resolved a two sigma uncertainty on an adjacent continuum region was used as an upper limit.

The DEM curves in Figs. 13 and 14 show the spline DEM values for points in the QS and AR respectively. Also included, below each corresponding spline plot, are plots of the ratio of the observed intensity to the theoretical intensity for each spectral line used to constrain the corresponding DEM curve. The ratio is plotted at the temperature of peak $G\left(T_{\mathrm{e}}, N_{\mathrm{e}}\right)$ for each line. The error bars on the ratio correspond to a combination of the one sigma uncertainties on the line fitting and an uncertainty 

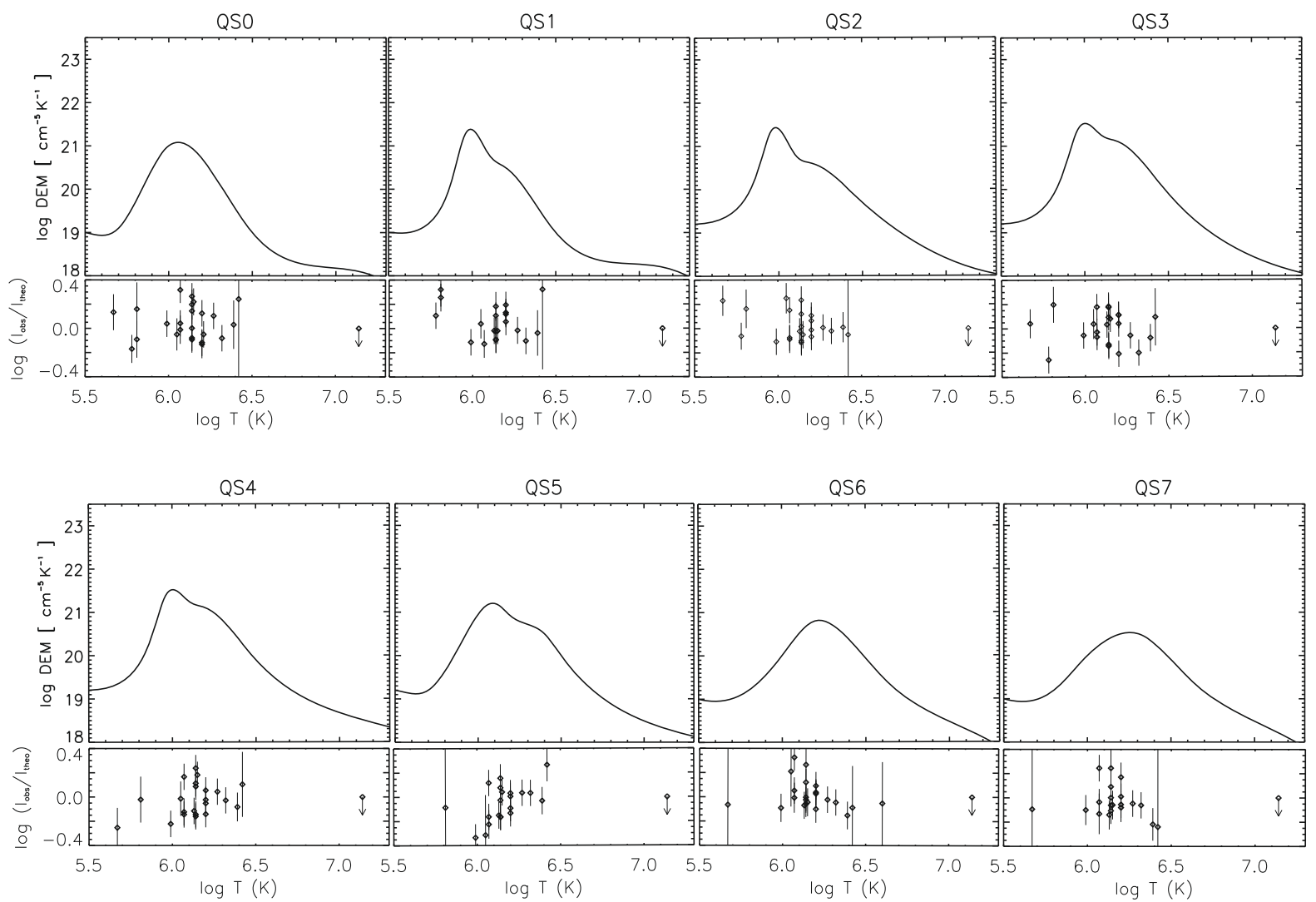

Fig. 13. EIS DEM curves for points in the quiet sun region shown in Fig. 3. Also included below each corresponding spline plot for each point in the quiet sun region are plots of the ratio of the observed intensity to the theoretical intensity for each spectral line used to constrain the corresponding DEM curve. The Fe XXIII $263.765 \AA$ line, indicated with an arrow, is included as an upper limit. The error bars on the ratio correspond to a combination of the one sigma uncertainties on the line fitting and an uncertainty of $22 \%$ on the observed intensity based on the pre-flight calibration of EIS (Lang et al. 2006).

of $22 \%$ on the observed intensity based on the pre-flight calibration of EIS (Lang et al. 2006).

The DEM curves for the Quiet Sun regions, QS0-QS7, shown in Fig. 13 are not isothermal, but have a relatively narrow distribution of temperatures (approximately $0.7 \mathrm{~K}$ on the $\log$ scale). The peak temperature varies between $\log T \sim$ 5.95-6.25, increasing with height above the limb. These Quiet Sun plots are included for comparison with Fig. 14, since they represent the background emission. These results are not in agreement with previous studies (e.g. Landi et al. 2002a,b) which found an isothermal plasma for the Quiet Sun. Some of the high temperature emission in our data for QS0-QS7 may be due to the proximity of the active region.

Figure 14 shows the DEM curves for points in the active region strip. For point AR0 the DEM curve is peaked around $\log T \sim 6.0$ and is similar to QS0. The DEM curve for point AR1 has a peak at $\log T \sim 6.0$ and a second peak at $\log T \sim$ 6.35 , with a higher temperature tail decreasing gradually. The $\mathrm{Fe} \mathrm{XVI} / \mathrm{Fe} \mathrm{XV}$ ratio is only sensitive to temperature above $\log T=6.2$ (Fig. 8), therefore no comparison can be made with the DEM curves derived for AR0 and AR1. The DEM curve for point AR2 remains approximately constant over the temperature range $\log T=5.9-6.4$ and peaks at a temperature of $\log T \sim 6.55$. For point AR3 the DEM curve remains approximately constant over the temperature range $\log T=5.7-6.5$ and peaks at a temperature of $\log T \sim 6.6$. The DEM curve for point AR4 has a broad peak around $\log T \sim 6.1$ and a higher peak at $\log T \sim 6.6$. The temperature derived from the Fe XVI/Fe XV ratio (Fig. 9) for AR2, AR3 and AR4 of $\log T=6.6$ is consistent with the peak in the DEM curves of $\log T=6.6$. For point AR5 the DEM curve peaks at $\log T \sim 6.1$ and $\log T \sim 6.5$, but falls off quite rapidly above $\log T=6.6$. The temperature derived from the Fe XVI/Fe XV ratio, Fig. 9, for AR5 also drops to $\log T=6.5$. The DEM curve for point AR6 remains approximately constant over the temperature range $\log T=6.0-6.5$, and falls off at higher temperatures. This is consistent with the temperature derived from the Fe XVI/Fe XV ratio. For point AR7 the DEM curve has a peak around $\log T \sim 6.1$ with a tail of higher temperature emission. This temperature is too low to be measured with the $\mathrm{Fe} \mathrm{XVI} / \mathrm{Fe} \mathrm{XV}$ ratio.

Overall, the high temperature peaks in the DEM curves derived for AR2-6 are consistent with temperature values derived from the Fe XVI to Fe XV ratio (Fig. 9).

The ratio of the emission measure value at $\log T=6.7$ to the value at $\log T=6.1$ was calculated at different distances from the active region core. The results are plotted in Fig. 15. The trend shown in the figure can be compared to the theoretical prediction in Fig. 7 in Patsourakos \& Klimchuk (2009). Although there are similarities, we find a sharper drop in this ratio with height. We note that the emission measure value at $\log T=7.15$ is about three orders of magnitude lower than the peak values, based on Fe XXIII upper limits.

XRT images of the active region were observed with the Al_poly, C_poly, Be_thin, Be_medium, Ti_poly, Al_thick and Be_thick filters as well as the filter combination Al_poly/Ti_poly. The data were processed using standard 

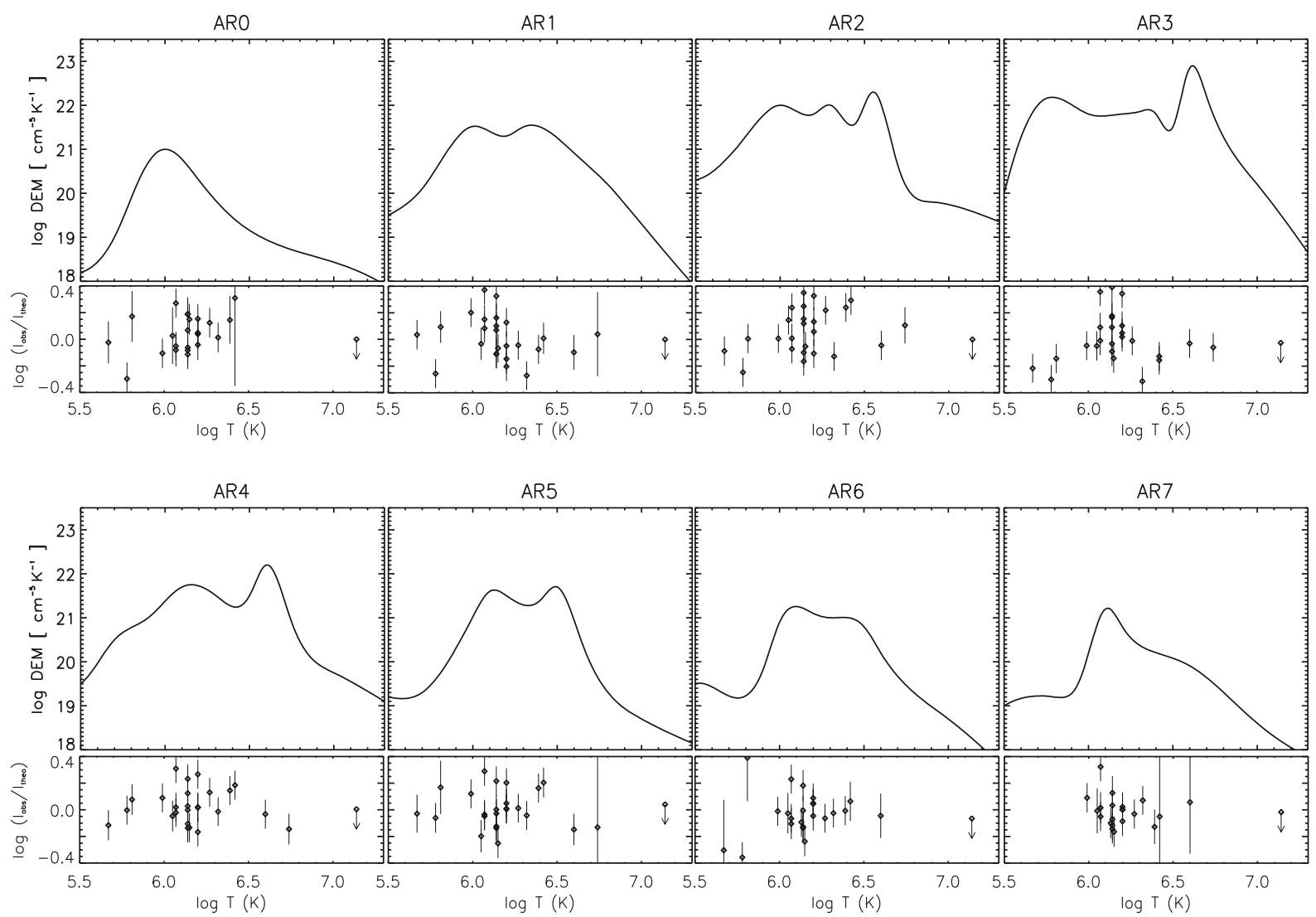

Fig. 14. Same as Fig. 13 but for the active region strip shown in Fig. 3.

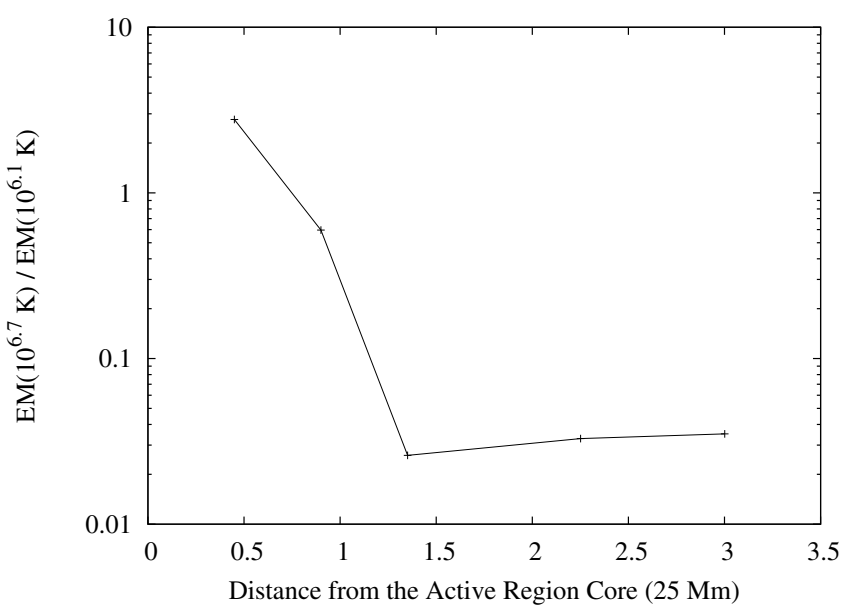

Fig. 15. The ratio of the emission measure value at $\log T=6.7$ to the value at $\log T=6.1$ plotted as a function of distance from the AR core.

XRT software available in SolarSoft (xrt_prep.pro). This includes the subtraction of a model dark frame, correction for vignetting, removal of high-frequency pattern noise, and normalisation by exposure time. Spacecraft jitter was removed using xrt_jitter.pro. Updated filter calibrations were used. The make_xrt_wave_resp.pro and make_xrt_temp_resp.pro routines were used to produce the spectral response and temperature response for each XRT channel. The xrt_dem_iterative2.pro routine available in SolarSoft was used to create DEM curves.

Figure 16 shows the location of an area above the limb over which intensities were summed from individual EIS spectral lines. This large area was chosen in order to increase the number of counts and to reduce any possible misalignments between the EIS and XRT data. The area corresponds to 726" squared. A corresponding DEM curve was created from EIS data and is displayed in Fig. 17. The Fe XXIII $263.765 \AA$ line, indicated with an arrow in the lower panel, is included as an upper limit.

The EIS raster across the area shown in Fig. 16 began at 11:51:04 UT and ended at 11:59:42 UT. During the same period XRT observations were made in each of the seven filters listed above, as well as the filter combination Al_poly/Ti_poly. Short and long exposure pairs of observations were taken for Al_poly, C_poly, Be_thin, Ti_poly and Al_poly/Ti_poly (see Table 1). The long exposure images for these filters were affected by saturation. As a result of the way saturated pixels are treated by the XRT software calculating average observed fluxes over the off-limb area using these images leads to the average values for the observed fluxes being underestimated. For this reason long and short exposures were co-aligned and combined. The combination of short and long exposures involved replacing the values of the observed flux for the saturated pixels in the long exposure images by the corresponding values in the short exposure images. This modification involved all four filters (Al_poly, C_poly, Be_thin, Ti_poly), as well as the filter combination Al_poly/Ti_poly, for which short/long pairs of observations were taken. Images taken with the other three filters (Be_thick, Al_thick, Be_med) were not affected by saturation. The intensities of these combined XRT images were summed over the off-limb area (see Table 1) and a corresponding DEM curve created. The solar coronal abundance values of Feldman et al. (1992) and the ionization equilibrium calculations of Mazzotta et al. (1998) were used in creating this DEM curve. The curve is overplotted in Fig. 17. The XRT DEM curve peaks around $\log T \sim 6.5$. 


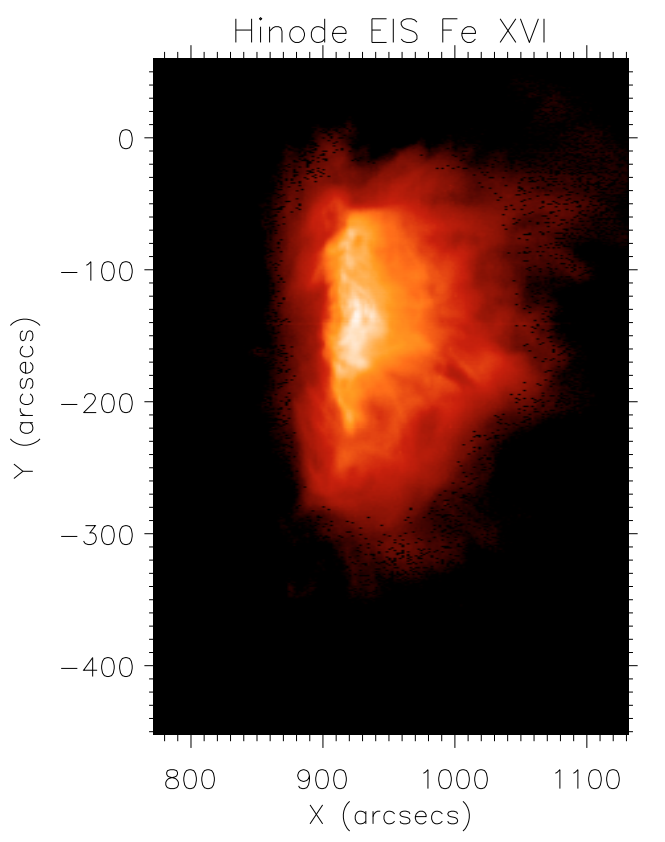

Fig. 16. EIS Fe XVI intensity map showing the location of the off-limb area in white.

The xrt_dem_iterative2.pro routine uses Monte Carlo iterations to estimate errors on the XRT DEM solution. For each iteration, the observed flux in each filter was varied randomly and the routine was run again with the new values. The distribution of these variations was Gaussian with a centroid equal to the observed flux and a width equal to the uncertainty (3\%). The solution sets from 200 different Monte Carlo realisations are overplotted in Fig. 18.

A lightcurve for the off-limb area created with images taken with the C_poly filter is shown in Fig. 19. A similar trend is observed in lightcurves for the other filters. The dashed lines in Fig. 19 indicate the beginning and end of the EIS raster across the off-limb area. The change in the C_poly intensity during this period is less than ten percent. This indicates that little variability was present in the off-limb area during this period.

In Fig. 17 the EIS DEM curve has a peak at $\log T \sim 6.6$, with a broader peak centered on $\log T \sim 6.15$. The EIS and XRT DEM curves appear to be in agreement in the temperature interval from $\log T=6.5-6.7$. Our EIS DEM curve is not well constrained above $\log T \sim 6.8$, the only constraint being the Fe XXIII 263.765 ̊ line included as an upper limit. We note the low XRT emission measure values below $\log T=6.2$ in Fig. 18 . Large variations were found between the solution sets from different Monte Carlo realisations at lower temperatures. It seems that the derived XRT DEM curve is unreliable below $\log T \sim 6.2$.

\section{Conclusions and discussion}

The EIS instrument on board Hinode provides an opportunity to study quantitatively the physical properties of the solar plasma over a range of temperatures simultaneously.

We have studied the electron density and temperature structure in an active region close to the solar limb. Using the line intensity ratio of Fe XII $186.887 \AA$ to $192.394 \AA$ the highest electron density values are found to be located in the core of the active region, with a peak value of $1.9 \times 10^{10} \mathrm{~cm}^{-3}$. The highest electron temperature values, $\log T \sim 6.7$, determined using line

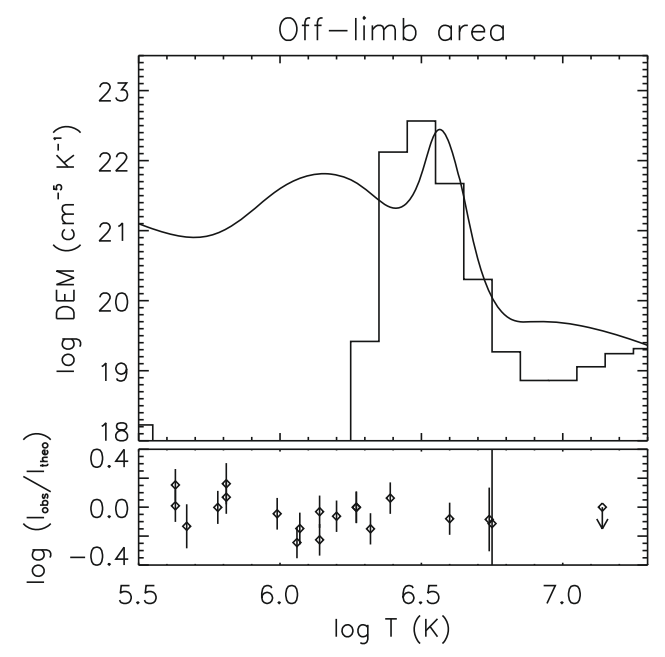

Fig. 17. Plot of the EIS (spline function) and XRT (histogram) DEM curves created for the off-limb area shown in Fig. 16. Also included is a plot of the ratio of the observed intensity to the theoretical intensity for each spectral line used to constrain the EIS DEM curve. The Fe XXIII 263.765 ̊̊ line, indicated with an arrow, is included as an upper limit. The error bars on the ratio correspond to a combination of the one sigma uncertainties on the line fitting and an uncertainty of $22 \%$ on the observed intensity based on the pre-flight calibration of EIS (Lang et al. 2006).

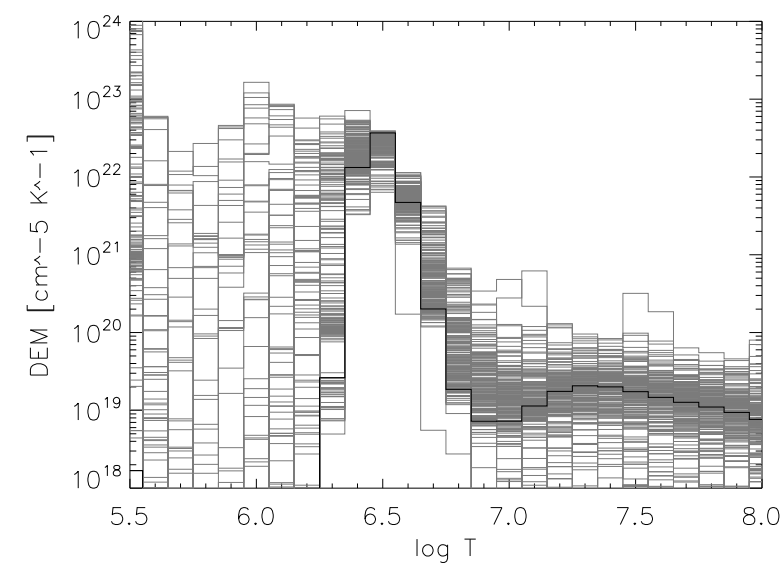

Fig. 18. DEM curves for the off-limb area of Fig. 16. Solution from xrt_dem_iterative2.pro in black. The solution sets from 200 different Monte Carlo realisations are overplotted.

ratios from adjacent ionization states of iron, are also found to be located in the core of the active region.

The emission measure (EM) loci technique and a differential emission measure (DEM) analysis were used to examine the thermal structure of the emitting plasma as a function of distance from the limb. The plasma along the line of sight in the active region was found to be multi-thermal for a sample of points at different distances from the limb.

By observing this active region "in profile" at the limb, we are able to examine the variation of height of its physical parameters. Consistent with the previous studies (Gabriel \& Jordan 1975; Vaiana 1976; Webb 1981; Mason et al. 1999; Del Zanna \& Mason 2003; Milligan et al. 2005; Tripathi et al. 2006), we have found the temperature of this active region to be highest in its core, $\log T \sim 6.6$, with lower temperatures at higher heights. Here in this analysis, we are able to go beyond the work of, e.g., Sterling (1997) and Sterling et al. (1997, 1999), which was primarily based on non-spatially-resolved observations. For 
Table 2. List of the strongest emission lines in the current study. Lines used in the DEM analysis are marked with asterisks.

\begin{tabular}{|c|c|c|c|c|}
\hline Ion & $\begin{array}{l}\lambda \\
\AA\end{array}$ & Transition & $\begin{array}{c}\log T_{\text {peak }} \\
\mathbf{K}\end{array}$ & Comment \\
\hline $\mathrm{OV}$ & 192.91 & $2 \mathrm{~s} 2 \mathrm{p}{ }^{3} \mathrm{P}_{2}-2 \mathrm{~s} 3 \mathrm{~d}^{3} \mathrm{D}_{2}$ & 5.4 & Blended with a Ca XVII line. \\
\hline $\mathrm{OV}$ & $248.46 *$ & $2 \mathrm{~s} 2 \mathrm{p}{ }^{1} \mathrm{P}_{1}-2 \mathrm{~s} 3 \mathrm{~s}^{1} \mathrm{~S}_{0}$ & 5.4 & \\
\hline $\mathrm{Mg} \mathrm{V}$ & $276.58 *$ & $2 \mathrm{~s}^{2} 2 \mathrm{p}^{4}{ }^{1} \mathrm{D}_{2}-2 \mathrm{~s} 2 \mathrm{p}^{5}{ }^{1} \mathrm{P}_{1}$ & 5.4 & Weak line. \\
\hline Mg VI (2) & $270.39 *$ & $\begin{array}{l}2 \mathrm{~s}^{2} 2 \mathrm{p}^{3}{ }^{2} \mathrm{D}_{5 / 2}-2 \mathrm{~s} 2 \mathrm{p}^{4}{ }^{2} \mathrm{P}_{3 / 2} \\
2 \mathrm{~s}^{2} 2 \mathrm{p}^{3}{ }^{2} \mathrm{D}_{3 / 2}-2 \mathrm{~s} 2 \mathrm{p}^{4}{ }^{2} \mathrm{P}_{3 / 2}\end{array}$ & 5.6 & Blended with an Fe XIV line. \\
\hline Mg VII & $278.4 *$ & $2 \mathrm{~s}^{2} 2 \mathrm{p}^{2}{ }^{3} \mathrm{P}_{2}-2 \mathrm{~s} 2 \mathrm{p}^{3}{ }^{3} \mathrm{~S}_{1}$ & 5.8 & Blended with a Si VII line. \\
\hline Mg VII & $280.74 *$ & $2 \mathrm{~s}^{2} 2 \mathrm{p}^{2}{ }^{1} \mathrm{D}_{2}-2 \mathrm{~s} 2 \mathrm{p}^{3}{ }^{1} \mathrm{P}_{1}$ & 5.8 & \\
\hline Si VII & $275.35 *$ & $2 \mathrm{~s}^{2} 2 \mathrm{p}^{4}{ }^{3} \mathrm{P}_{2}-2 \mathrm{~s} 2 \mathrm{p}^{5}{ }^{3} \mathrm{P}_{2}$ & 5.8 & \\
\hline $\mathrm{Fe} X$ & $184.54 *$ & $3 s^{2} 3 p^{5}{ }^{2} P_{3 / 2}-3 s^{2} 3 p^{4}\left({ }^{1} D\right) 3 d^{2} S_{1 / 2}$ & 6.0 & \\
\hline Fe XI & $180.43 *$ & $3 s^{2} 3 p^{4}{ }^{3} P_{2}-3 s^{2} 3 p^{3}\left({ }^{4} S\right) 3 d^{3} D_{3}$ & 6.1 & Blended with an $\mathrm{Fe} \mathrm{X}$ line. \\
\hline Fe XI & 182.17 & $3 s^{2} 3 p^{4}{ }^{3} P_{1}-3 s^{2} 3 p^{3}\left({ }^{4} S\right) 3 d^{3} D_{2}$ & 6.1 & \\
\hline Fe XI & $188.22 *$ & $3 s^{2} 3 p^{4}{ }^{3} P_{2}-3 s^{2} 3 p^{3}\left({ }^{2} D\right) 3 d^{3} P_{2}$ & 6.1 & \\
\hline Fe XI & 188.3 & $3 s^{2} 3 p^{4}{ }^{3} P_{2}-3 s^{2} 3 p^{3}\left({ }^{2} \mathrm{D}\right) 3 d^{1} P_{1}$ & 6.1 & \\
\hline Fe XI & 192.81 & $3 s^{2} 3 p^{4}{ }^{3} \mathrm{P}_{1}-3 s^{2} 3 p^{3}\left({ }^{2} \mathrm{D}\right) 3 d^{3} \mathrm{P}_{2}$ & 6.1 & Blended with a Ca XVII line. \\
\hline Fe XII & $192.39 *$ & $3 s^{2} 3 p^{3}{ }^{4} S_{3 / 2}-3 s^{2} 3 p^{2}\left({ }^{3} P\right) 3 d^{4} P_{1 / 2}$ & 6.1 & \\
\hline Fe XII & 193.51 & $3 s^{2} 3 p^{3}{ }^{4} S_{3 / 2}-3 s^{2} 3 p^{2}\left({ }^{3} P\right) 3 d^{4} P_{3 / 2}$ & 6.1 & \\
\hline Fe XII & 196.64 & $3 s^{2} 3 p^{3}{ }^{2} D_{5 / 2}-3 s^{2} 3 p^{2}\left({ }^{1} D\right) 3 d^{2} D_{5 / 2}$ & 6.1 & Blended with an Fe VIII line. \\
\hline Fe XII & 203.73 & $3 \mathrm{~s}^{2} 3 \mathrm{p}^{3}{ }^{2} \mathrm{D}_{5 / 2}-3 \mathrm{~s}^{2} 3 \mathrm{p}^{2}\left({ }^{1} \mathrm{~S}\right) 3 \mathrm{~d}^{2} \mathrm{D}_{5 / 2}$ & 6.1 & Blended with two Fe XIII lines. \\
\hline Si IX & 258.08 & $2 \mathrm{~s}^{2} 2 \mathrm{p}^{2}{ }^{1} \mathrm{D}_{2}-2 \mathrm{~s} 2 \mathrm{p}^{3}{ }^{1} \mathrm{D}_{2}$ & 6.1 & \\
\hline Si X & 258.37 & $2 \mathrm{~s}^{2} 2 \mathrm{p}^{2} \mathrm{P}_{3 / 2}-2 \mathrm{~s} 2 \mathrm{p}^{2}{ }^{2} \mathrm{P}_{3 / 2}$ & 6.1 & \\
\hline Si X & $261.06 *$ & $2 \mathrm{~s}^{2} 2 \mathrm{p}^{2} \mathrm{P}_{3 / 2}-2 \mathrm{~s} 2 \mathrm{p}^{2}{ }^{2} \mathrm{P}_{1 / 2}$ & 6.1 & \\
\hline Si X & 271.99 & $2 s^{2} 2 p^{2} \mathrm{P}_{1 / 2}-2 \mathrm{~s} 2 \mathrm{p}^{2}{ }^{2} \mathrm{~S}_{1 / 2}$ & 6.1 & \\
\hline S X & 264.23 & $2 \mathrm{~s}^{2} 2 \mathrm{p}^{3}{ }^{4} \mathrm{~S}_{3 / 2}-2 \mathrm{~s} 2 \mathrm{p}^{4}{ }^{4} \mathrm{P}_{5 / 2}$ & 6.15 & \\
\hline Fe XIII & 196.53 & $3 \mathrm{~s}^{2} 3 \mathrm{p}^{2}{ }^{1} \mathrm{D}_{2}-3 \mathrm{~s}^{2} 3 \mathrm{p} 3 \mathrm{~d}^{2} \mathrm{~F}_{3}$ & 6.2 & \\
\hline Fe XIII & 201.12 & $3 s^{2} 3 p^{2}{ }^{3} P_{1}-3 s^{2} 3 p 3 d^{3} D_{1}$ & 6.2 & \\
\hline $\mathrm{Fe}$ XIII & $202.04 *$ & $3 s^{2} 3 p^{2}{ }^{3} P_{0}-3 s^{2} 3 p 3 d^{3} P_{1}$ & 6.2 & \\
\hline Fe XIII (2) & 203.83 & $\begin{array}{l}3 s^{2} 3 p^{2}{ }^{3} P_{2}-3 s^{2} 3 p 3 d^{3} D_{3} \\
3 s^{2} 3 p^{2}{ }^{3} P_{2}-3 s^{2} 3 p 3 d^{3} D_{2}\end{array}$ & 6.2 & Blended with an Fe XII line. \\
\hline Fe XIV & $264.79 *$ & $3 s^{2} 3 p^{2} \mathrm{P}_{3 / 2}-3 s 3 p^{2}{ }^{2} \mathrm{P}_{3 / 2}$ & 6.3 & \\
\hline Fe XIV & $274.2 *$ & $3 s^{2} 3 p^{2} \mathrm{P}_{1 / 2}-3 \mathrm{~s} 3 \mathrm{p}^{2}{ }^{2} \mathrm{~S}_{1 / 2}$ & 6.3 & Blended with Si VII and Ni XV lines. \\
\hline $\mathrm{Fe} X \mathrm{XV}$ & $284.16 *$ & $3 \mathrm{~s}^{2}{ }^{1} \mathrm{~S}_{0}-3 \mathrm{~s} 3 \mathrm{p}^{1} \mathrm{P}_{1}$ & 6.3 & Blended with Al IX, Fe XVII and Ni XV lines. \\
\hline Fe XVI & $262.98 *$ & $3 p^{2} P_{3 / 2}-3 d^{2} D_{5 / 2}$ & 6.4 & \\
\hline Fe XVI & 265.01 & $3 \mathrm{p}^{2} \mathrm{P}_{3 / 2}-3 \mathrm{~d}^{2} \mathrm{D}_{3 / 2}$ & 6.4 & Weak line. \\
\hline $\mathrm{Ca} \mathrm{XV}$ & $200.98 *$ & $2 \mathrm{~s}^{2} 2 \mathrm{p}^{2}{ }^{3} \mathrm{P}_{0}-2 \mathrm{~s} 2 \mathrm{p}^{3}{ }^{3} \mathrm{D}_{1}$ & 6.6 & \\
\hline Fe XVII & $275.55 *$ & $2 s^{2} 2 p^{5} 3 p{ }^{1} P_{1}-2 s^{2} 2 p^{5} 3 d^{1} D_{2}$ & 6.6 & \\
\hline Ca XVII & $192.82 *$ & $2 \mathrm{~s}^{2}{ }^{1} \mathrm{~S}_{0}-2 \mathrm{~s} 2 \mathrm{p}{ }^{1} \mathrm{P}_{1}$ & 6.7 & Blended with $\mathrm{Fe} \mathrm{XI}$ and $\mathrm{O} \mathrm{V}$ lines. \\
\hline Fe XXIII & $263.77 *$ & $2 \mathrm{~s}^{2}{ }^{1} \mathrm{~S}_{0}-2 \mathrm{~s} 2 \mathrm{p}{ }^{3} \mathrm{P}_{1}$ & 7.15 & \\
\hline Fe XXIV & 192.03 & $1 s^{2} 2 s^{2} S_{1 / 2}-1 s^{2} 2 p^{2} P_{3 / 2}$ & 7.25 & Blended with an Fe XI line. \\
\hline
\end{tabular}

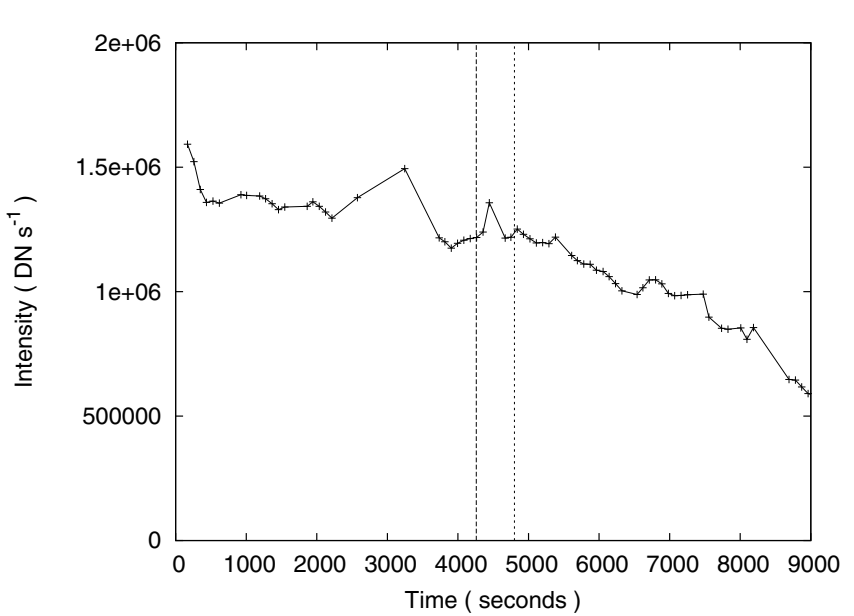

Fig. 19. Lightcurve for the off-limb area in Fig. 16 created from images taken with the XRT C_poly filter. The dashed lines indicate the beginning and end of the EIS raster across this area.

example, we have found that the hottest locations occur in concentrated regions inside the core of the active region.
Schmelz et al. (2009a,b) and Reale et al. (2009) have analysed Hinode/XRT data for active regions. Both sets of authors use broad-band observations to derive emission measure curves. Their analyses apparently provide clear evidence for the presence of plasma around $10 \mathrm{MK}$. With our Hinode/EIS analysis we were unable to definitely identify the Fe XXIII $263.765 \AA$ line (temperature of formation $\log T \sim 7.15$ ) at any location in the active region. We note that value of our EIS DEM curve at $\log T=7.15$ is larger than the value of the XRT DEM curve obtained by Schmelz et al. (2009b) using a manual forward-folding routine at the same temperature. Our EIS DEM curve is not well constrained above $\log T \sim 6.8$, the only constraint being an upper limit on the Fe XXIII $263.765 \AA$ line. Our EIS and XRT DEM analyses appear to be in agreement in the temperature interval from $\log T=6.5-6.7$.

Our findings of localized hot regions inside of the AR are also consistent with other spatially-resolved analyses of active regions (e.g., Yoshida \& Tsuneta 1996; Tripathi et al. 2006). Here we expanded upon these earlier works by doing a quantitative examination with the full imaging and spectral capabilities of EIS. Our results are in qualitative agreement with those of Patsourakos \& Klimchuk (2009), but further studies are needed. 
In summary, these new observations and results demonstrate the full capability of Hinode/EIS to derive electron density, temperature and DEM distributions. As solar activity increases we hope to obtain more observations to confirm our results.

Acknowledgements. B.O.D., G.D.Z., H.E.M. and D.T. acknowledge STFC. B.O.D. was supported by funding from the Gates Cambridge Trust. A.C.S. was supported by funding from NASA's SR\&T and LWS programs. We would like to thank Mark Weber and the rest of XRT team for their advice on creating XRT DEM curves. We would also like to thank the anonymous referee for constructive comments which have improved the quality of this manuscript. Hinode is a Japanese mission developed and launched by ISAS/JAXA, collaborating with NAOJ as a domestic partner, NASA and STFC (UK) as international partners. Scientific operation of the Hinode mission is conducted by the Hinode science team organized at ISAS/JAXA. This team mainly consists of scientists from institutes in the partner countries. Support for the post-launch operation is provided by JAXA and NAOJ (Japan), STFC (UK), NASA, ESA, and NSC (Norway).

\section{References}

Aschwanden, M. J., Schrijver, C. J., \& Alexander, D. 2001, ApJ, 550, 1036 Cargill, P. J. 1994, ApJ, 422, 381

Cargill, P. J., \& Klimchuk, J. A. 1997, ApJ, 478, 799

Culhane, J. L., Harra, L. K., James, A. M., et al. 2007, Sol. Phys., 243, 19

Del Zanna, G. 1999, Ph.D. Thesis, Univ. of Central Lancashire

Del Zanna, G., \& Mason, H. E. 2003, A\&A, 406, 1089

Del Zanna, G., \& Ishikawa, Y. 2009, A\&A, 508, 1517

Del Zanna, G., Landini, M., \& Mason, H. E. 2002, A\&A, 385, 968

Dere, K. P., Landi, E., Mason, H. E., Monsignori Fossi, B. C., \& Young, P. R. 1997, A\&AS, 125, 149

Feldman, U., Mandelbaum, P., Seely, J. F., Doschek, G. A., \& Gursky, H. 1992 ApJS, 81, 387

Freeland, S. L., \& Handy, B. N. 1998, Sol. Phys., 182, 497

Gabriel, A. H., \& Jordan, C. 1975, MNRAS, 173, 397

Golub, L., Deluca, E., Austin, G., et al. 2007, Sol. Phys., 243, 63

Haugan, S. V. H. 1997, The Component Fitting System (CFIT) for IDL, CDS software note No. 47

Klimchuk, J. A. 2006, Sol. Phys., 234, 41

Klimchuk, J. A., Patsourakos, S., \& Cargill, P. J. 2008, ApJ, 682, 1351

Ko, Y.-K., Doschek, G. A., Warren, H. P., \& Young, P. R. 2009, ApJ, 697, 1956

Kosugi, T., Matsuzaki, K., Sakao, T., et al. 2007, Sol. Phys., 243, 3
Landi, E., Feldman, U., \& Dere, K. P. 2002a, ApJ, 574, 495

Landi, E., Feldman, U., \& Dere, K. P. 2002b, ApJS, 139, 281

Landi, E., Del Zanna, G., Young, P. R., et al. 2006, ApJS, 162, 261

Lang, J., Kent, B. J., Paustian, W., et al. 2006, Appl. Opt., 45, 8689

Liang, G. Y., Whiteford, A. D., \& Badnell, N. R. 2009, A\&A, 500, 1263

Mason, H. E., Landi, E., Pike, C. D., \& Young, P. R. 1999, Sol. Phys., 189, 129

Mazzotta, P., Mazzitelli, G., Colafrancesco, S., \& Vittorio, N. 1998, A\&AS, 133, 403

Milligan, R. O., Gallagher, P. T., Mathioudakis, M., Keenan, F. P., \& Bloomfield, D. S. 2005, MNRAS, 363, 259

Monsignori Fossi, B. C., \& Landini, M. 1991, Adv. Space Res., 11, 281

Parker, E. N. 1988, ApJ, 330, 474

Patsourakos, S., \& Klimchuk, J. A. 2009, ApJ, 696, 760

Pye, J. P., Evans, K. D., Hutcheon, R. J., et al. 1978, A\&A, 65, 123

Reale, F., Testa, P., Klimchuk, J. A., \& Parenti, S. 2009, ApJ, 698, 756

Schadee, A., de Jager, C., \& Svestka, Z. 1983, Sol. Phys., 89, 287

Schmelz, J. T., Saar, S. H., DeLuca, E. E., et al. 2009a, ApJ, 693, L131

Schmelz, J. T., Kashyap, V. L., Saar, S. H., et al. 2009b, ApJ, 704, 863

Sterling, A. C. 1997, Geophys. Res. Lett., 24, 2263

Sterling, A. C., Hudson, H. S., \& Watanabe, T. 1997, ApJ, 479, L149

Sterling, A. C., Pike, C. D., Mason, H. E., Watanabe, T., \& Antiochos, S. K. 1999, ApJ, 524, 1096

Storey, P. J., \& Zeippen, C. J. 2010, A\&A, 511, A78

Storey, P. J., Del Zanna, G., Mason, H. E., \& Zeippen, C. J. 2005, A\&A, 433, 717

Tripathi, D., Mason, H. E., \& Young, P. R. 2006, in ESA SP, SOHO-17, 10 Years of SOHO and Beyond, 617

Tripathi, D., Mason, H. E., Young, P. R., Chifor, C., \& Del Zanna, G. 2008a, in First Results From Hinode, ed. S. A. Matthews, J. M. Davis, \& L. K. Harra, ASP Conf. Ser., 397, 45

Tripathi, D., Mason, H. E., Young, P. R., \& Del Zanna, G. 2008b, A\&A, 481, L53

Tripathi, D., Mason, H. E., Dwivedi, B. N., del Zanna, G., \& Young, P. R. 2009, ApJ, 694, 1256

Vaiana, G. S. 1976, Royal Soc. London Philos. Trans. Ser. A, 281, 365

Warren, H. P., \& Winebarger, A. R. 2003, ApJ, 596, L113

Watanabe, T., Hara, H., Shimizu, T., et al. 1995, Sol. Phys., 157, 169

Webb, D. F. 1981, in Solar Active Regions: A monograph from Skylab Solar Workshop III, ed. F. Q. Orrall, 165

Yoshida, T., \& Tsuneta, S. 1996, ApJ, 459, 342

Young, P. R., Del Zanna, G., Mason, H. E., et al. 2007, PASJ, 59, 857

Young, P. R., Watanabe, T., Hara, H., \& Mariska, J. T. 2009, A\&A, 495, 587 